\title{
Morphologic and cytogenetic differences between post-polycythemic myelofibrosis and primary myelofibrosis in fibrotic stage
}

\author{
Leonardo Boiocchi ${ }^{1,2,4}$, Susan Mathew ${ }^{1}$, Umberto Gianelli ${ }^{2}$, Alessandra Iurlo ${ }^{3}$, \\ Tommaso Radice ${ }^{3}$, Sharon Barouk-Fox ${ }^{1}$, Daniel M Knowles ${ }^{1}$ and Attilio Orazi ${ }^{1}$
}

${ }^{1}$ Department of Pathology and Laboratory Medicine, Weill Cornell Medical College/New York Presbyterian Hospital, New York, NY, USA; ${ }^{2}$ Division of Pathology, Department of Pathophysiology and Transplantation, University of Milan Medical School, IRCCS Ca' Granda-Ospedale Maggiore Policlinico Foundation, Milan, Italy and ${ }^{3}$ Hematology and Transplantation Unit, IRCCS Ca' Granda-Ospedale Maggiore Policlinico Foundation, Milan, Italy

Polycythemia vera and primary myelofibrosis share a propensity to progress toward a myelofibrotic late stage with overlapping clinical characteristics. Bone marrow features potentially useful for distinguishing the two entities have not been thoroughly investigated and, currently, clinical history is used for purposes of disease classification. This study describes in detail the morphologic features of 23 cases of post-polycythemic myelofibrosis and 15 cases of primary myelofibrosis with a similar degree of fibrosis, from two large medical centers. Cytogenetic results were available in 19 post-polycythemic myelofibrosis and in 13 primary myelofibrosis cases. JAK2 status and follow-up information was available in all cases. Cellularity was increased in both groups, but more so in post-polycythemic myelofibrosis than in primary myelofibrosis. In post-polycythemic myelofibrosis, most megakaryocytes retained polycythemia vera-like features including normally folded and/or hyperlobulated nuclei devoid of severe maturation defects; only in a few cases were rare tight clusters present. In primary myelofibrosis cases, megakaryocytes showed pronounced anomalies, including increased nuclear:cytoplasmic ratio, abnormal clumping of chromatin and frequent tight clustering. No differences in blast number $(<1 \%)$ or in the myeloid:erythroid ratio were observed. Post-polycythemic myelofibrosis showed a higher degree of karyotypic alterations and higher percentage of cases with complex karyotype and/or two or more clones. Chromosome 1 defects were common in post-polycythemic myelofibrosis, whereas isolated del(20q) was the most common alteration in primary myelofibrosis. No survival differences were noted between the two groups. Post-polycythemic myelofibrosis cases retain a distinct megakaryocytic morphology that represents a useful clue for differential diagnosis. In addition, they more often display a complex karyotype than do primary myelofibrosis cases. These results suggest that myelofibrosis in polycythemia vera represents a form of progression characterized by profound genetic damage whereas in primary myelofibrosis it is an intrinsic part of the phenotypic manifestation of the disease, not necessarily associated with adverse cytogenetics.

Modern Pathology (2013) 26, 1577-1585; doi:10.1038/modpathol.2013.109; published online 21 June 2013

Keywords: bone marrow biopsy; cytogenetics; myeloproliferative neoplasms; primary myelofibrosis; post-polycythemic myelofibrosis

Correspondence: Dr A Orazi, MD, FRCPath, Department of Pathology and Laboratory Medicine, Weill Cornell Medical College, 525 East 68th Street, New York, NY 10065, USA.

E-mail: ato9002@med.cornell.edu

${ }^{4}$ Current address: Pathology Section, Department of Molecular and Translational Medicine, University of Brescia, Brescia, Italy. This work has been presented in part as an oral platform at the 101th Annual Meeting of the United States and Canadian Academy of Pathology, Vancouver, BC, Canada, 17-23 March 2012.

Received 27 March 2013; revised 3 May 2013; accepted 3 May 2013; published online 21 June 2013
Philadelphia chromosome-negative myeloproliferative neoplasms all share a propensity to progress toward a late myelofibrotic stage characterized by splenomegaly and decreased peripheral blood counts because of marrow failure. Post-polycythemic myelofibrosis occurs in approximately $20 \%$ of the polycythemic patients after 10 years or more from diagnosis. Post-polycythemic myelofibrosis and fibrotic primary myelofibrosis have overlapping clinical characteristics (eg, splenomegaly and 
decreased peripheral blood counts). Conversely, post-essential thrombocythemia myelofibrosis is much less common, occurring in $<1 \%$ of cases at 10 years and $<10 \%$ at 15 years follow-up.

Differential diagnosis between post-polycythemic (and post-essential thrombocythemia) myelofibrosis and primary myelofibrosis relies almost entirely on clinical criteria. Bone marrow features potentially useful to discriminate between these diseases having not been much investigated. The current WHO 2008 classification requires evidence of a previous diagnosis of polycythemia vera (or essential thrombocythemia) for purposes of diagnosing these cases of secondary myelofibrosis. ${ }^{1}$

Polycythemia vera consists of an erythrocytotic phase with effective proliferation in the erythroid, granulocytic and megakaryocytic lineages and a distinctively panmyelotic hypercellular bone marrow; in this phase peripheral blood shows a mild to overt excess of red blood cells and hemoglobin with reduced EPO levels. After 10-15 years from the onset, $20 \%$ of polycythemia vera cases evolve in a post-polycythemic myelofibrosis also known as 'spent phase' characterized by bone marrow fibrosis with collagen deposition, decreasing blood counts and worsening splenomegaly. ${ }^{1}$

In post-polycythemic myelofibrosis, bone marrow morphologic features share similarities with those of advanced stage primary myelofibrosis (MF-2/3). ${ }^{2}$ However, in spite of the fact that both post-polycythemic myelofibrosis and primary myelofibrosis similarly share severe marrow fibrosis as the dominant morphologic characteristic, the underlying pathogenetic mechanisms leading to post-polycythemic myelofibrosis and primary myelofibrosis are most likely profoundly different. Post-polycythemic myelofibrosis represents a late evolution of a slow evolving clonal disease in which many genetic alterations have progressively accumulated and lead over time to a transformation from a 'proliferative' neoplasm to a marrow failure syndrome, whereas primary myelofibrosis in comparison shows a propensity to fibrosis deposition from a much earlier phases of disease and has a shorter clinical course. ${ }^{2}$ Currently, therapeutic approaches in patients with a myeloproliferative neoplasm in advanced fibrotic stages are similar, irrespective of the specific entity. ${ }^{3}$ However, it is not clear whether there are differences between these two conditions, for example, in relation to bone marrow and cytogenetic findings. Therefore, this study aims to describe the morphologic, immunophenotypic and cytogenetic features of post-polycythemic myelofibrosis and primary myelofibrosis with similar degrees of fibrosis, in an attempt to identify novel findings, which could complement those already in use, ${ }^{1}$ helping to discriminate these two distinct clinicopathological entities. Comprehension of these mechanisms could possibly open new therapeutic horizons for these patients, with the introduction of novel target therapies, as is happening in many other hematological and solid neoplasms.

\section{Materials and methods}

\section{Patients}

Twenty-three patients with diagnosis of postpolycythemic myelofibrosis and 15 patients with primary myelofibrosis in fibrotic phase (MF-2 and MF-3) were included in the study. Cases of postpolycythemic myelofibrosis were identified from the files of two reference centers, Weill Cornell Medical College (NY; 15 cases) and IRCCS Ca' Granda-Ospedale Maggiore Policlinico Foundation (Milan, Italy; 8 cases) in a period spanning from 2008 to 2010. All cases of primary myelofibrosis were obtained from Weill Cornell Medical College files. Cases were selected from the original reports and all slides were reviewed by $\mathrm{AO}, \mathrm{LB}$ and UG. Diagnosis was confirmed according to 2008 WHO criteria. ${ }^{1} \mathrm{~A}$ bone marrow biopsy taken at the fibrotic stage and detailed clinical history were available for review in all cases. Mutational status of JAK2 was known in all cases and conventional cytogenetic results were available for most. The design of this study was approved by the Institutional Review Board of Weill Cornell Medical College and the Ethical Committee of the IRCCS Ca' GrandaOspedale Maggiore Policlinico Foundation.

\section{Morphologic and Immunohistochemical Analysis}

Bone marrow biopsies from Weill Cornell Medical College were fixed in Bouin's solution, decalcified in a nitric acid solution and embedded in paraffin; biopsies from Ospedale Maggiore Policlinico were fixed in neutral buffered formalin, decalcified in ethylenediaminetetraacetic acid and embedded in paraffin. Sections from each case were stained with hematoxylin and eosin (H\&E) and Giemsa staining for morphologic examination and with Gomori's silver impregnation and Masson's trichrome staining to evaluate fibrosis. Reticulin and trichrome histochemical staining was performed using an automated stainer (Leica Microsystems, Bannockburn, IL, USA) and the manufacturer's specific kits and protocols with only minor modifications. Morphologic variables that were evaluated included: absolute cellularity (percentage), age-adjusted cellularity (classified as decreased, normal or increased), myeloid/erythroid ratio (M:E) and percentage of blasts in the biopsy, evaluated using immunohistochemistry for CD34. Particular attention was paid to megakaryocytic morphology: presence of tight clusters, dwarf forms, naked nuclei and quantification of the proportion of megakaryocytes with 'polycythemia vera-like' or 'primary myelofibrosis-like' features in the two diseases were noted for each 
case. We defined megakaryocytes as 'polycythemia vera-like' or 'primary myelofibrosis-like' according to the description given in the 2008 WHO classification of the typical megakaryocytes characterizing these two myeloproliferative neoplasms. Therefore, we defined as 'polycythemia vera-like' megakaryocytes those displaying a variable degree of pleomorphism, with normally folded or deeply lobulated nuclei, lacking significant cytologic abnormality, whereas 'primary myelofibrosis-like' megakaryocytes were those showing deviation from normal nuclear:cytoplasmic ratio, abnormal patterns of chromatin clumping, with bulbous, 'cloud-like' or 'balloon-shaped' nuclei., ${ }^{1,2}$ As a general rule, to be classified as 'primary myelofibrosis-like', megakaryocytes had to be more atypical than 'polycythemia vera-like' megakaryocytes. Gomori's staining was used to evaluate stromal reticulin deposition and Masson's trichrome staining was useful to quantify collagen deposition. The grade of bone marrow fibrosis was assessed applying the European Consensus Grading System of marrow fibrosis, a four-tier grading scale ranging from MF-0 to MF-3. ${ }^{4}$ All morphological evaluations were performed by AO, LB and UG, blinded to diagnosis and clinical data. Pathologists examined the slides separately and exchanged results. All discrepant evaluations were then discussed and reconciled. Consensus was reached in all cases.

\section{Immunohistochemistry}

Immunohistochemical studies were performed using a Leica BOND III autostainer (Leica Microsystems, Buffalo Grove, IL, USA). Monoclonal antibodies against the following antigens were used: CD42b (dilution 1:100; mouse anti-human; LeicaNovocastra, USA), glycophorin C (dilution 1:50; mouse anti-human; Abcam, USA), myeloperoxidase (dilution 1:5000, polyclonal rabbit anti- human; Dako, USA), CD34 (dilution 1:50, mouse antihuman, BioGenex, USA) and CD117 (dilution 1:75, polyclonal rabbit anti- human; Dako). Antigen retrieval was obtained by using Bond Epitope Retrieval Solution 2 (for CD34, CD42b and CD117) and solution 2 (for 30' for glycophorin C and myeloperoxidase) and heat-induced epitope retrieval at $99-100^{\circ} \mathrm{C}$ for $20^{\prime}$ (for solution 2) and for 30' (for solution 1) on the Bond system (Leica Microsystems). An external negative control and a positive control were stained in each case.

\section{Molecular Analysis}

Genomic DNA was extracted from peripheral whole blood or from purified granulocytes using standard manual methods. ${ }^{5}$ The JAK2 V617F mutation was detected by means of allele-specific polymerase chain reaction according to the protocol of Baxter et $a l^{6}$ The presence of the mutation status was confirmed by direct sequencing (ABI PRISM 310 Genetic Analyzer, Applied Biosystems, Warrington, England) using the Big Dye Terminator Cycle Sequencing Kit (Applied Biosystems).

\section{Cytogenetic Analysis}

Cells collected from bone marrow aspirates taken with bone marrow biopsy were cultured unstimulated for $24 \mathrm{~h}$ in MarrowMax medium (Gibco, Life Technologies, Carlsbad, CA, USA) and harvested according to standard protocols. G-banded metaphases were analyzed and described according to the International System for Human Cytogenetic Nomenclature (ISCN 2009). ${ }^{7}$

\section{Follow-Up}

Follow-up data were available for all patients. Duration of disease was calculated as the interval from the first diagnosis of polycythemia vera in polycythemic phase or primary myelofibrosis to the biopsy of post-polycythemic myelofibrosis or primary myelofibrosis in fibrotic phase; overall survival was defined as the time from the first diagnosis to the last contact with patient; survival after the onset of fibrosis as the interval from the diagnosis of post-polycythemic myelofibrosis or primary myelofibrosis in fibrotic phase to the last contact with the patient. In case of the death of a patient, the specific cause was recorded.

\section{Statistical Analysis}

Statistical analysis of results was performed with the SPSS statistics 17 software (SPSS, Chicago, IL, USA). For all statistical tests, a $P$-value $<0.05$ was considered significant; all tests were two-sided. Wilcoxon signed-rank test was used to evaluate categorical variables such as reticulin fibrosis, ageadjusted cellularity and number of megakaryocytes. McNemar's test was used to evaluate differences in binomial variables as presence of clusters, maturational defects, bulbous or naked nuclei in megakaryocytes. $T$-test was applied to evaluate differences in continuous variables as hemoglobin, white blood cells and platelets counts, mean corpuscular volume, absolute cellularity and myeloid/erythroid ratio. Pearson $\chi^{2}$ test was used to evaluate differences in proportions. Overall survival in the two groups was described by Kaplan-Meier curves, and differences evaluated with the log-rank test.

\section{Results}

\section{Patients}

In the post-polycythemic myelofibrosis group, 15 patients $(65 \%)$ were men and 8 were women 
$(35 \%)$, with a mean age of 64.3 years (median $=64$ years); in the primary myelofibrosis group, 9 patients were men $(60 \%)$ and 6 were women $(40 \%)$, with a mean age of 60.4 years (median $=60$ years) (Table 1). All cases were reviewed and classified according to current 2008 WHO criteria. Hemoglobin concentration at the time of diagnosis of post-polycythemic myelofibrosis was found to be higher than in primary myelofibrosis patients with corresponding marrow fibrosis (mean: 11.9 vs $9.5 \mathrm{~g} / \mathrm{l}$ respectively; $P=0.002$ ); however, hemoglobin was below diagnostic values during the polycythemic phase of polycythemia vera and all patients had already suspended phlebotomies. White blood cell count was found to be higher in the postpolycythemic myelofibrosis group than in primary myelofibrosis patients (14.3 vs $\left.8.4 \times 10^{9} / 1 ; P=0.02\right)$; no significant differences were observed between the two groups for platelet count or mean corpuscular volume. Splenomegaly was a common finding in both groups. All clinical data for both groups are summarized in Table 1.

\section{Morphologic Analysis}

All patients in both groups had increased cellularity for their age; however, absolute cellularity was found to be higher in post-polycythemic myelofibrotic patients (mean: 95\%) than in primary myelofibrosis patients (mean: 66\%; $P<0.001$ ). Among post-polycythemic myelofibrosis cases, fibrosis in 10 patients $(43 \%)$ was graded as MF-2 and as MF-3 in the remaining $13(57 \%)$; among primary myelofibrosis cases, $6(40 \%)$ were graded as MF-2, the remaining $9(60 \%)$ as MF-3 (Table 2). Comparison of frequencies of MF-2 and MF-3 fibrosis in the postpolycythemic myelofibrosis and primary myelofi-

Table 1 Summary of patients' clinical data

\begin{tabular}{|c|c|c|}
\hline & $\begin{array}{c}\text { Post-PV MF } \\
(\text { tot }=23)\end{array}$ & $\begin{array}{c}P M F \\
(\text { tot }=15)\end{array}$ \\
\hline Age (mean) & 64.3 & 60.4 \\
\hline $\operatorname{Sex}(M / F)$ & $15 / 8$ & $9 / 6$ \\
\hline Hemoglobin (g/l) & 11.9 & 9.5 \\
\hline MCV (fL; mean) & 84.4 & 82.9 \\
\hline WBC ( × $10^{9} / \mathrm{l} ;$ mean $)$ & 14.3 & 8.4 \\
\hline Platelets ( $\times 10^{9} / 1$; mean $)$ & 288 & 220 \\
\hline Splenomegaly (yes/no) & $17 / 3$ & $8 / 1$ \\
\hline JAK2V617 (mutated/WT) & $23 / 0(100 \%)$ & $9 / 6(60 \%)$ \\
\hline $\begin{array}{l}\text { Mean follow-up } \\
\text { (months; range) }\end{array}$ & $163(72-264)$ & $36(0-60)$ \\
\hline Therapy & $\begin{array}{c}\text { Phlebotomy }(23)^{\mathrm{a}} \text {; } \\
\text { hydroxyurea (14); } \\
\text { decitabine (1); } \\
\text { anagrelide and } \\
\text { interferon (1) }\end{array}$ & $\begin{array}{c}\text { None (9); prednisone } \\
\text { and thalidomide (1); } \\
\text { anagrelide (1); } \\
\text { darbopoietin (1); } \\
\text { interferon (1) }\end{array}$ \\
\hline
\end{tabular}

Abbreviations: MCV, median corpuscular volume; post-PV MF, post-polycythemic myelofibrosis; PMF, primary myelofibrosis; WBC, white blood cells

${ }^{a}$ Already suspended at time of diagnosis of post-PV MF. brosis groups showed no significant difference $(P=0.83)$. Patterns of distribution of reticulin and/ or collagen fibrosis showed no recognizable differences in the two groups (Figure 1).

In post-polycythemic myelofibrosis cases, the majority of megakaryocytes (81\%) retained 'polycythemia vera-like' features: they maintained normally folded or hyperlobulated nuclei devoid of severe maturational defects. In post-polycythemic myelofibrosis, 'primary myelofibrosis-like' megakaryocytes with an abnormal pattern of chromatin clumping and other pronounced atypical features were relatively rare $(19 \%)$. Tight clusters were not observed in 10 cases, they were only occasionally seen $(<2$ in the whole-bone marrow biopsy) in 5 cases and were numerous in the remaining 8 cases (Figure 2). In contrast, in primary myelofibrosis cases the majority of megakaryocytes $(76 \%)$ showed 'primary myelofibrosis-like' features, the most striking being maturational defects such as increased nuclear:cytoplasmic ratio or abnormally clumped chromatin. The remaining megakaryocytes (24\%) showed a lesser degree of atypia and overlap with those seen in cases of post-polycythemic myelofibrosis. The extensive presence of tight clusters of megakaryocytes was a constant finding in all primary myelofibrosis cases. Frequencies of 'polycythemia vera-like' and 'primary myelofibrosis-like' megakaryocytes in the two groups were significantly different: 'polycythemia vera-like' megakaryocytes prevailed in post-polycythemic myelofibrosis ( $81 \%$ vs $24 \%$ of primary myelofibrosis, $P<0.001$ ), whereas 'primary myelofibrosis-like' features characterized primary myelofibrosis cases but were only rarely observed in post-polycythemic myelofibrosis cases $(76 \%$ vs $19 \%$, respectively; $P<0.001$; Table 2).

No differences in CD34 + blast number $(<1 \%$ in all cases) or in myeloid/erythroid ratio were observed between the two groups $(\mathrm{M}: \mathrm{E}=4.2$ in post-polycythemic myelofibrosis vs $\mathrm{M}: \mathrm{E}=4.4$ in primary myelofibrosis).

Table 2 Summary of bone marrow morphologic features including bone marrow cellularity, myeloid/erythroid ratio, fibrosis grade, frequency of 'polycythemia vera-like' and 'primary myelofibrosislike' megakaryocytes and CD34+ blasts in patients with post-polycythemic myelofibrosis and patients with primary myelofibrosis

\begin{tabular}{lccc}
\hline & $\begin{array}{c}\text { Post-PV MF } \\
\text { (tot=23) }\end{array}$ & $\begin{array}{c}\text { PMF } \\
\text { (tot=15) }\end{array}$ & P-value \\
\hline Cellularity (mean) & $95 \%$ & $66 \%$ & $<0.001$ \\
Myeloid/erythroid ratio & 4.2 & 4.4 & $\mathrm{NS}$ \\
MF-2/MF-3 (ratio) & $10 / 13(0.77)$ & $6 / 9(0.66)$ & $\mathrm{NS}$ \\
'PV-like' megakaryocytes (\%) & $81 \%$ & $24 \%$ & $<0.001$ \\
'PMF-like' megakaryocytes (\%) & $19 \%$ & $76 \%$ & $<0.001$ \\
CD34+ blasts (\%) & $<1 \%$ & $<1 \%$ & $\mathrm{NS}$
\end{tabular}

Abbreviations: NS, not significative; post-PV MF, post-polycythemic myelofibrosis; PMF, primary myelofibrosis; PV, polycythemia vera. 
Reticulin
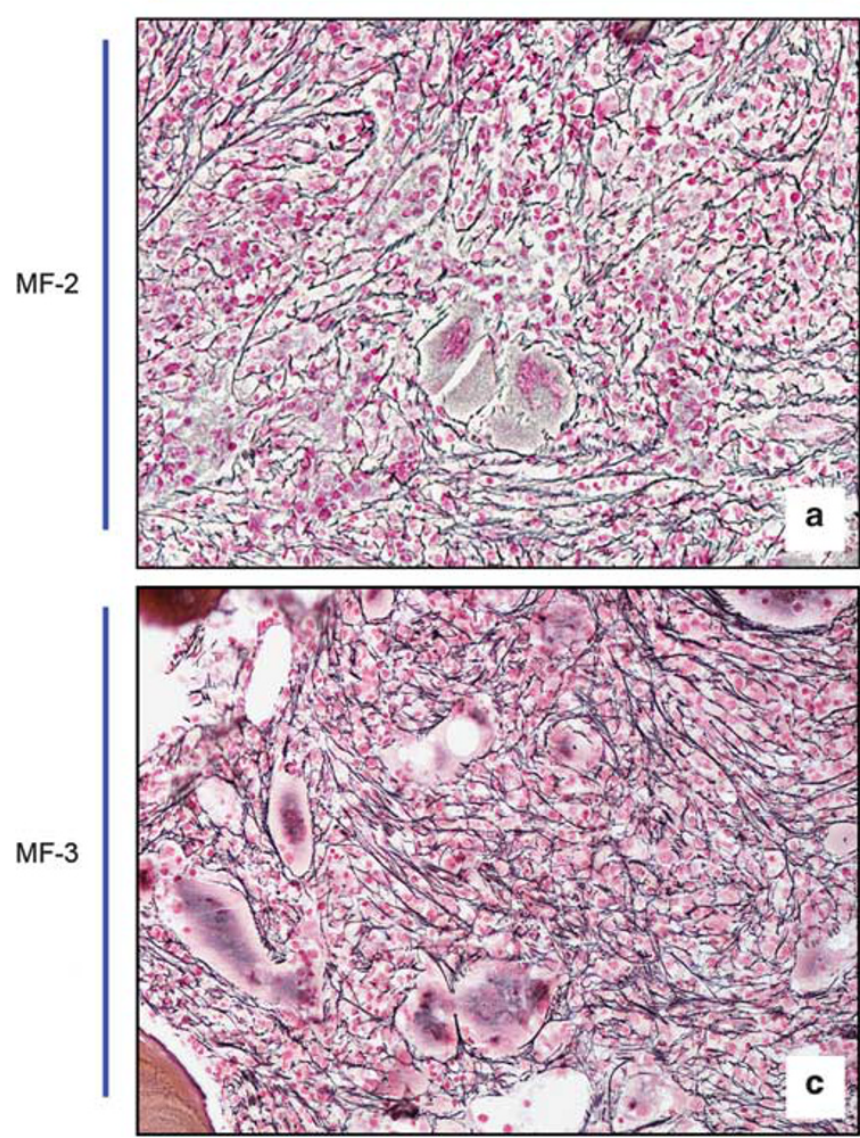

Trichrome
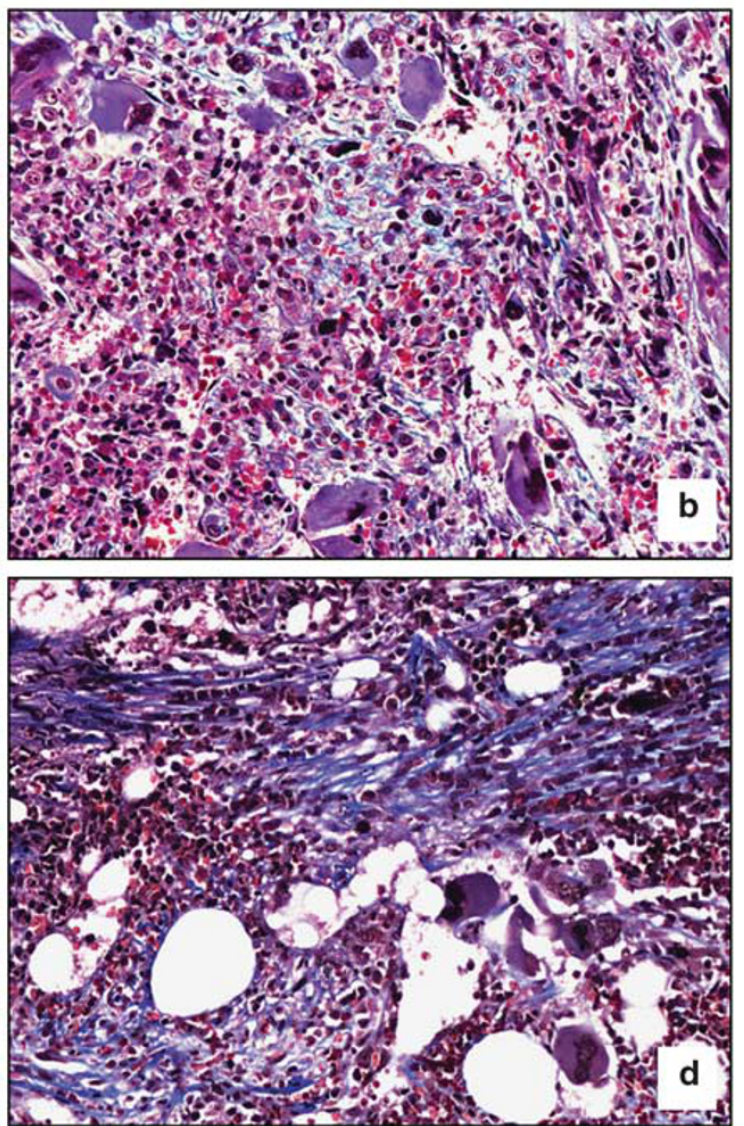

Figure 1 Fibrosis grade MF-2, defined as a diffuse and dense increase in reticulin with extensive intersections, occasionally with only focal bundles of collagen and/or focal osteosclerosis; and MF-3, a diffuse and dense increase in reticulin with extensive intersections, with coarse bundles of collagen, often associated with significant osteosclerosis are required for the diagnosis of post-polycythemic myelofibrosis and primary myelofibrosis in fibrotic stages. The pattern of fibrosis in both conditions is overlapping, without any specific pattern for the two entities $(\mathbf{a}, \mathbf{c})$ reticulin staining, $\times 20$ magnification; (b, d) trichrome staining, $\times 20$ magnification.

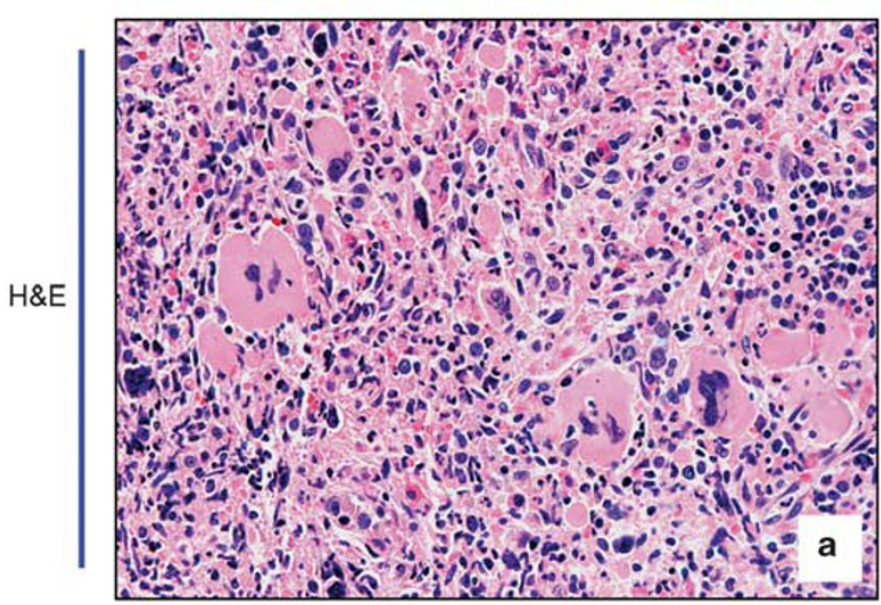

PMF

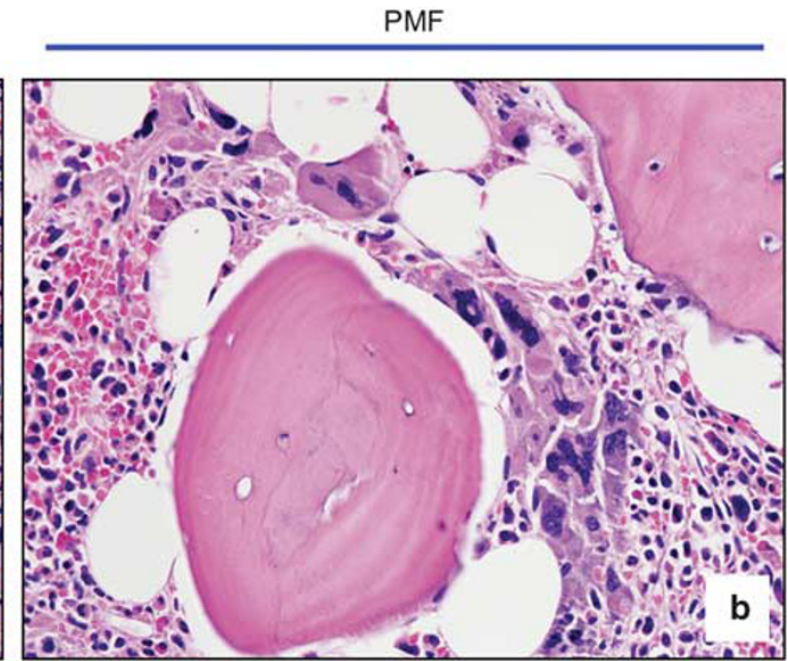

Figure 2 (a) In post-polycythemic myelofibrosis cases the majority of megakaryocytes retained 'polycythemia vera-like' features such as normally folded or hyperlobulated nuclei, and were devoid of severe maturational defects; rare tight clusters could be observed in a minority of cases (H\&E, $\times 20$ magnifications). (b) In primary myelofibrosis cases the majority of megakaryocytes display 'primary myelofibrosis-like' features, the most striking being maturational defects such as increased nuclear/cytoplasm ratio, abnormally clumped chromatin and diffuse formation of tight clusters. In general, megakaryocytes are more atypical than those seen in polycythemia vera (H\&E, $\times 20$ magnifications). 


\section{Molecular Analysis}

All 23 patients (100\%) with post-polycythemic myelofibrosis and 9 patients with primary myelofibrosis $(60 \%)$ carried JAK2V617F mutation.

\section{Cytogenetic Analysis}

Karyotype cytogenetics results were available for 19 of $23(82 \%)$ post-polycythemic myelofibrosis cases and for 13 of $15(87 \%)$ cases of primary myelofibrosis. Results for all cases are reported in Table 3.
In some cases, aspiration of bone marrow resulted in a dry tap, and it was not possible to collect and culture enough viable bone marrow cells to perform the karyotypic analysis.

Seven cases of $19(37 \%)$ of post-polycythemic myelofibrosis showed a normal karyotype. In the other 12 cases (63\%), alterations and defects were numerous, including either structural or numerical abnormalities, such as chromosome gain or loss, translocations with presence of derivative chromosomes and partial deletions. The presence of three or more cytogenetic anomalies defined a complex

Table 3 Cytogenetic results for all patients with post-polycythemic myelofibrosis $(n=19)$ and patients with primary myelofibrosis $(n=13)$

\begin{tabular}{|c|c|c|}
\hline Case \# & Diagnosis & Cytogenetics \\
\hline PV 1 & Post-PV MF & NA \\
\hline PV 2 & Post-PV MF & 46,XX,-14,der(17)t(14;17)(q11.2;q21),der(20)t(17;20)(q21;q13.1), + mar1[17]/46,XX,-20, + mar2[2]/46,XX[1] \\
\hline PV 3 & Post-PV MF & 46,XY,?add(1)(p36.1)[11]/46,Y,add(X)(p22.1),-11, + mar[4]/46,XY[5] \\
\hline PV 4 & Post-PV MF & Normal \\
\hline PV 5 & Post-PV MF & 46,XY,del(20)(q11.2)[14]/45,X,-Y,add(3)(q29),add(4)(q?21),add(7)(p11.2)[cp5] \\
\hline PV 6 & Post-PV MF & 45,XX,t(9;12)(q22;p13)[13],der(13)t(1;13)(q12;p11.2)[18],-18[20],add(20)(q11.2)[cp20]. \\
\hline PV 7 & Post-PV MF & NA \\
\hline PV 8 & Post-PV MF & 47,XY, + 9,del(20)(q11.2)[2]/48,idem, + 8[10]/47,idem,der(22)t(1;22)(q12;p12)[5]/46,XY[2] \\
\hline PV 9 & Post-PV MF & $47, \mathrm{XX},+8[15] / 46, \mathrm{XX}[4]$ \\
\hline PV 10 & Post-PV MF & $46, \mathrm{XX}, \operatorname{del}(7)(\mathrm{q} 11.2 \mathrm{q} 22)[3] / 46, \mathrm{XX}[17]$ \\
\hline PV 11 & Post-PV MF & NA \\
\hline PV 12 & Post-PV MF & Normal \\
\hline PV 13 & Post-PV MF & NA \\
\hline PV 14 & Post-PV MF & $47, \mathrm{XY},+\operatorname{der}(1 ; 9)(q 10 ; \mathrm{p} 10)[17] / 47, \mathrm{XY},+9[2]$ \\
\hline PV 15 & Post-PV MF & Normal \\
\hline PV 16 & Post-PV MF & 46,XY,del(20)(q12) [3]/46,XY [17] \\
\hline PV 17 & Post-PV MF & Normal \\
\hline PV 18 & Post-PV MF & $46, \mathrm{XY},+1, \operatorname{der}(1 ; 7)(\mathrm{q} 10 ; \mathrm{p} 10)[20] / 46, \mathrm{XY}, \operatorname{del}(20)(\mathrm{q} 11)[2]$ \\
\hline PV 19 & Post-PV MF & $46, X Y,+1, \operatorname{der}(1 ; 15)(q 10 ; q 10)[13] / 46, X Y[16]$ \\
\hline PV 20 & Post-PV MF & Normal \\
\hline PV 21 & Post-PV MF & $47, \mathrm{XY}, \mathrm{t}(1 ; 19)(\mathrm{q} 23 ; \mathrm{p} 13.3),+\operatorname{der}(19) \mathrm{t}(1 ; 19)(\mathrm{q} 23 ; \mathrm{p} 13.3)[14] / 46, \mathrm{XY}[7]$ \\
\hline PV 22 & Post-PV MF & Normal \\
\hline PV 23 & Post-PV MF & Normal \\
\hline PMF 1 & PMF & 46,XY,del(13)(q12q14)[9]/46,idem,del(9)(q13q22)[3]/46,XY,del(20)(q11.2q13.1)[2]/46,XY[6] \\
\hline PMF 2 & PMF & Normal \\
\hline PMF 3 & PMF & Normal \\
\hline PMF 4 & PMF & 46,XY,del(20)(q11.2)[15]/46,XY[5] \\
\hline PMF 5 & PMF & $48, \mathrm{XX},+8,+9[1] / 48, \mathrm{XX},+8,+\operatorname{der}(9) \mathrm{t}(1 ; 9)(\mathrm{q} 11 ; \mathrm{q} 12)[8] / 96, \mathrm{XXXX},+8 \mathrm{x} 2,+\operatorname{der}(9) \mathrm{x} 2[4] / 46, \mathrm{XX}[4] / 92, \mathrm{XXXX}[3]$ \\
\hline PMF 6 & PMF & 46,XX,-6, + mar[4]/45,XX,der(7)add(7)(p22)add(7)(q22),-20[3]/46,XX[12] \\
\hline PMF 7 & PMF & 46,XY,del(20)(q11.2)[6]/46,XY[1] \\
\hline PMF 8 & PMF & Normal \\
\hline PMF 9 & PMF & Normal \\
\hline PMF 10 & PMF & Normal \\
\hline PMF 11 & PMF & 46,XX,del(20)(q11.2q13.1)[17]/46,XX[3] \\
\hline PMF 12 & PMF & NA \\
\hline PMF 13 & PMF & Normal \\
\hline PMF 14 & PMF & 46,XY,del(7)(q22), del(20) (q11.2q13.1)[20] \\
\hline
\end{tabular}

Abbreviations: NA, not available; post-PV MF, post-polycythemic myelofibrosis; PMF: primary myelofibrosis. 
karyotype. Among 12 cases that presented alterations, 6 showed a complex karyotype $(50 \%$ of cases with alterations; $32 \%$ of all post-polycythemic myelofibrosis cases with cytogenetic results) whereas the remaining 6 showed $<3$ clonal anomalies $(50 \% ; 32 \%)$. Cases with follow-up karyotype(s) showed a progressive cytogenetic complexity with acquisition of new abnormalities not present in the initial diagnostic karyotype. Five cases $(42 \%$; 26\%) presented only one clone with alterations, whereas the remaining $7(58 \% ; 37 \%)$ showed two or more clones (see Table 4). In particular, post-polycythemic myelofibrosis patient 8 showed different clones with a clear progressive accumulation of alterations. Among structural alterations, balanced and unbalanced translocations were common, including $t(1 ; 13), t(1 ; 19), t(9 ; 12), t(14 ; 17), t(17 ; 20)$, although none of these was a recurrent abnormality. Numeric alterations were not main features of karyotypes in post-polycythemic myelofibrosis cases: 45 to 47 chromosomes were found in all cases, with exception of patient 10, which showed a clone with 48 chromosomes. Only trisomy of chromosome 8 recurred in two cases. Seven cases of post polycythemic myelofibrosis showed alterations of chromosome 1, either numerical or structural. Philadelphia chromosome was never found.

Six of $13(46 \%)$ primary myelofibrosis cases showed a normal karyotype. Among the remaining seven showing alterations (54\%), four cases $(58 \%$; $31 \%$ of all primary myelofibrosis cases with cytogenetic results) showed less than three clonal chromosomal abnormalities; three cases (42\%; 23\%)

Table 4 Cytogenetic results: frequencies of chromosomal alterations and number of clones in patients with post-polycythemic myelofibrosis and patients with primary myelofibrosis

\begin{tabular}{|c|c|c|}
\hline & $\begin{array}{l}\text { Post-PV MF } \\
\quad(\text { tot }=19)\end{array}$ & $\begin{array}{c}P M F \\
\text { (tot }=13)\end{array}$ \\
\hline \multicolumn{3}{|c|}{ Among all cases with cytogenetics } \\
\hline \multicolumn{3}{|l|}{ Normal/altered } \\
\hline Normal (\%) & $7(37 \%)$ & $6(46 \%)$ \\
\hline With alterations (\%) & $12(63 \%)$ & $7(54 \%)$ \\
\hline \multicolumn{3}{|l|}{$\begin{array}{l}\text { Among those with alterations } \\
\text { ( } \% \text { of cases with alterations; }\end{array}$} \\
\hline \multicolumn{3}{|l|}{$\%$ of cases with cytogenetic) } \\
\hline \multicolumn{3}{|c|}{ Number of alterations (per clone) } \\
\hline$<3$ Alterations (\%) & $6(50 \% ; 32 \%)$ & $4(58 \% ; 31 \%)$ \\
\hline Complex karyotype (\%) & $6(50 \% ; 32 \%)$ & $3(42 \% ; 23 \%)$ \\
\hline \multicolumn{3}{|l|}{ Number of clones } \\
\hline 1 Clone (\%) & $5(42 \% ; 26 \%)$ & $4(58 \% ; 31 \%)$ \\
\hline$\geq 2$ Clones $(\%)$ & $7(58 \% ; 37 \%)$ & $3(42 \% ; 23 \%)$ \\
\hline $\begin{array}{l}\text { Complex karyotype and } \\
\text { multiple clones }(\geq 2)\end{array}$ & $7(58 \% ; 37 \%)$ & $3(42 \% ; 23 \%)$ \\
\hline
\end{tabular}

Abbreviations: post-PV MF, post-polycythemic myelofibrosis; PMF, primary myelofibrosis.

Distribution of cases in classes defined by the number of karyotypic alterations or of clones shown in karyotype was described both as percentual fractions of the total number of cases included in each group (post-PV MF =19; PMF =13), and as fraction of only cases with alterations in each group (post-PV $\mathrm{MF}=12 ; \mathrm{PMF}=7$ ). showed a complex karyotype. Four cases (58\%; $31 \%$ ) showed only one pathologic clone, whereas in the remaining three cases $(42 \% ; 23 \%)$ two or more clones were present (see Table 4). Among cases with only one alteration, del(20q) was seen in three cases $(42 \%$; $23 \%)$; moreover, one case $(8 \%$, patient 14$)$ showed one clone with del(20q) associated with $\operatorname{del}(7 q)$. Only one unbalanced translocation, $\operatorname{der}(9) t(1 ; 9)$, was observed among primary myelofibrosis cases.

Among primary myelofibrosis cases numeric alterations were found only in two cases (patient 6 and patient 5). Philadelphia chromosome was never identified among primary myelofibrosis cases.

\section{Survival}

Mean duration of follow-up in patients with postpolycythemic myelofibrosis was 163 months (range: 72-264 months) and in primary myelofibrosis patients was 36 months (range: 0-60 months). Only two patients, one in each group, died from disease progression with bone marrow failure. However, when comparing the two groups, no significant difference in overall survival was found.

\section{Discussion}

Morphological differences among the Philadelphiachromosome BCR/ABL1-negative myeloproliferative neoplasms in fibrotic stage have not been extensively studied and current WHO diagnostic criteria $^{1}$ for post-polycythemic and post-essential thrombocythemia myelofibrosis require a degree of fibrosis, MF-2 or MF-3, which overlap what seen in the overt fibrotic stage of primary myelofibrosis. Besides documentation of a previous diagnosis of polycythemia, the diagnosis of post-polycythemic myelofibrosis vera requires the presence of overt reticulin and collagen fibrosis, anemia, leukoerythroblastic blood picture, splenomegaly and/or selected constitutional symptoms. In an attempt to identify new parameters useful in separating post-polycythemic myelofibrosis from primary myelofibrosis with a similar degree of fibrosis, we compared bone marrow morphological features, JAK2 mutational status and cytogenetics results in patients belonging to these two diagnostic groups.

Our results show that there are morphologic differences. In particular, the majority of megakaryocytes in the fibrotic stage of polycythemia vera still retain the typical morphology seen in the polycythemic phase, that is, pleomorphic megakaryocytes with no, or only occasional nuclear maturational defects without significant megakaryocytic atypia (no abnormal chromatin clumping or other significant nuclear abnormalities) or alterations in the nuclear:cytoplasmic ratio. On the contrary, evident atypical changes in megakaryocytes such 
as the presence of abnormal nuclear:cytoplasmic ratio together with the occurrence of tight clusters, are the hallmarks of primary myelofibrosis in fibrotic stage (MF-2/3).

$J A K 2 V 617 F$ mutation was found in all our post-polycythemic myelofibrosis patients, but in only $60 \%$ of primary myelofibrosis patients. Similar frequency of $J A K 2$ mutations in post-polycythemic myelofibrosis has also been recently reported in another study. ${ }^{8}$ In addition, high $J A K 2 V 617 F$ allele burden was documented in these cases when compared with post-essential thrombocythemia cases. Another study had previously associated higher JAK2V617F allele burden during the polycythemic phase of polycythemia vera with increased risk of progression toward a fibrotic phase. ${ }^{9}$ Both studies, however, did not find any association between $J A K 2 V 617 F$ allele burden and risk of leukemic transformation. Unluckily, data on $J A K 2 \mathrm{~V} 617 \mathrm{~F}$ allele burden were not available for our cases of post-polycythemic myelofibrosis patients.

Karyotypic analysis with conventional methods has already been shown to have an important prognostic role in myeloproliferative neoplasms in fibrotic phase and, in some cases at least, can also influence the therapeutic approach to treat these patients. Presence of a complex karyotype has been associated with a poorer prognosis, whereas isolated $\operatorname{del}(20 q)$ or del(13q) have a less adverse impact. ${ }^{10}$ Presence of del(5q) can be a predictive marker of response to treatment with lenalidomide. ${ }^{11}$

In spite of the extensive marrow fibrosis, for most of the cases included in this study it was possible to obtain enough viable cells from bone marrow aspiration to perform cytogenetic analysis. The frequency and number of alterations was higher in the post-polycythemic myelofibrosis than in the primary myelofibrosis group; the former also showed a higher frequency of complex karyotypes. Among these only t $(9 ; 12)$ was balanced, whereas all the others were unbalanced. Despite the presence of numerous different structural alterations, the number of chromosomes in both groups was well preserved, without any marked aneuploidy. All alterations we found in post-polycythemic myelofibrosis patients, with the exception of $+8,+9$ and $-\mathrm{Y}$, are not among those commonly described as recurrent in the polycythemic phase of the disease. ${ }^{12,13}$ Seven cases of post-polycythemic myelofibrosis showed alterations of chromosome 1 . Their frequency, however, was not as high as previously reported in the literature. ${ }^{14}$

The high frequency of both structural and numerical alterations in post-polycythemic myelofibrosis, often with the presence of multiple clones, suggests an increased fragility of chromosomes and defects in mechanisms that relates to the correct duplication and rearrangement of chromosomes during mitosis. Predominance of structural defects over numerical alterations could also suggest that the machinery that controls the correct segregation of chromosomes during the cell cycle is still relatively well preserved even at this late stage of disease progression.

Complex karyotypes were less common in primary myelofibrosis cases than in post-polycythemic myelofibrosis. In particular, chromosome 1 abnormalities were more common in post-polycythemic myelofibrosis when compared with primary myelofibrosis ( $36 \%$ vs $8 \%$ of all cases). In the comparison among the two groups not only the percentage of cases with two or more clones, but also the cumulative percentage of cases showing a complex karyotype and/or 2 or more clones was inferior in primary myelofibrosis than in post-polycythemic myelofibrosis ( $43 \%$ vs $58 \%$ ).

However, alterations highlighted by conventional cytogenetic analysis are a visible manifestation of molecular damage. The mechanisms that lead to this damage are far from being explained, at least in the setting of Philadelphia-negative myeloproliferative neoplasms. A recent study based on a high-resolution SNP array ${ }^{15}$ provides some evidence of specific genomic alterations associated with progression of polycythemia vera toward a fibrotic phase and with leukemic evolution. However, for most polycythemia vera patients the mechanism that leads to the progressive accumulation and to the karyotypic complexity during the course of their disease remains unknown. It has been speculated that a possible important role for abnormalities affecting the mechanism that govern and eventually correct errors in the replication and the recombination of the genetic material over those that survey the correct segregation of chromosomes.

An interesting aspect of our findings is that polycythemia vera and primary myelofibrosis, although both characterized by the progressive and irreversible deposition of reticulin and collagen fibrosis, most likely follow different paths of genetic damage. More than two-thirds of primary myelofibrosis patients, in fibrotic stage (MF-2/MF-3) did not show cytogenetic alterations or carried del(20q) as the only one; in one case del(20q) was associated with del(7q) another alteration commonly found in primary myelofibrosis and other myeloid neoplasms.

Thus, it can be hypothesized that post-polycythemic myelofibrosis truly represents a manifestation of disease progression to a terminal stage of the disease, whereas this is not necessarily true in primary myelofibrosis, a disease in which myelofibrosis is intrinsically related to the pathogenesis of the disease, developing relatively early in its natural course without evidence of major cytogenetic damage. It is important to note that none of the patients in our studies received cytotoxic therapy (other than hydroxyurea) so it seems that the explanation of these differences cannot be due to effects of different therapies.

In conclusion, our attempt at identifying parameters that allow distinguishing post-polycythemic myelofibrosis and primary myelofibrosis in fibrotic 
phase with increasing accuracy could prospectively have an important role in improving the prognosis of these diseases. Today, these patients still have a life expectation of only a few years. ${ }^{16,17}$ Even without new target therapies ready for clinical use, with the exception of JAK2 inhibitors, ${ }^{18}$ a better pathological classification will give the possibility of optimizing the existing therapeutic regimens for Philadelphia-negative myeloproliferative neoplasms. This approach has already given good results for primary myelofibrosis. ${ }^{19}$

In the future, it will be important to investigate and dissect the precise molecular mechanisms that lead to the dramatic bone marrow alterations and that at last determine the fatal evolution of these neoplasms. This process, as a result, will allow acting even more precisely and effectively with new therapeutic tools that, from futuristic prospects, are becoming reality in many other types of tumors.

\section{Disclosure/conflict of interest}

The authors declare no conflict of interest.

\section{References}

1 Thiele J, Kvasnicka HM, Orazi A, et al. Polycythaemia vera. In: Swerdlow SH, Campo E, Harris NL, et al. (eds). WHO Classification of Tumours of Haematopoietic and Lymphoid Tissues. IARC: Lyon, France, 2008, pp 40-43.

2 Thiele J, Kvasnicka HM, Tefferi A, et al. Primary myelofibrosis. In: Swerdlow SH, Campo E, Harris NL, et al. (eds). WHO Classification of Tumours of Haematopoietic and Lymphoid Tissues. IARC: Lyon, France, 2008, pp 44-47.

3 Reilly JT, McMullin MF, Beer PA, et al. Guideline for the diagnosis and management of myelofibrosis. Br J Haematol 2012;158:453-471.

4 Thiele J, Kvasnicka HM, Facchetti F, et al. European consensus on grading bone marrow fibrosis and assessment of cellularity. Haematologica 2005;90:1128-1132.

5 Sambrook J, Fritsch EF, Maniatis T. Molecular Cloning: A Laboratory Manual. Cold Spring Harbor Laboratory Press: New York, NY, USA, 1989.

6 Baxter EJ, Scott LM, Campbell PJ, et al. Acquired mutation of the tyrosine kinase JAK2 in human myeloproliferative disorders. Lancet 2005;365: 1054-1061.

7 Shaffer LG, Slovak ML, Campbell LJ (eds) ISCN (2009): An International system for Human Cytogenetic Nomenclature. Karger: Basel, 2009.

8 Helbig G, Wieczorkiewicz A, Wozniczka K, et al. The JAK2V617F tyrosine kinase mutation has no impact on overall survival and the risk of leukemic transformation in myelofibrosis. Med Oncol 2012;29: 2379-2384.

9 Passamonti F, Rumi E, Pietra D, et al. A prospective study of 338 patients with polycythemia vera: the impact of JAK2 (V617F) allele burden and leukocytosis on fibrotic or leukemic disease transformation and vascular complications. Leukemia 2010;24:1574-1579.

10 Hussein K, Van Dyke DL, Tefferi A. Conventional cytogenetics in myelofibrosis: literature review and discussion. Eur J Haematol 2009;82:329-338.

11 Santana-Davila R, Tefferi A, Holtan SG, et al. Primary myelofibrosis is the most frequent myeloproliferative neoplasm associated with $\operatorname{del}(5 \mathrm{q})$ : clinicopathologic comparison of del(5q)-positive and -negative cases. Leuk Res 2008;32:1927-1930.

12 Andrieux JL, Demory JL. Karyotype and molecular cytogenetic studies in polycythemia vera. Curr Hematol Rep 2005;4:224-229.

13 Westwood NB, Gruszka-Westwood AM, Pearson CE, et al. The incidences of trisomy 8, trisomy 9 and D20S108 deletion in polycythaemia vera: an analysis of blood granulocytes using interphase fluorescence in situ hybridization. Br J Haematol 2000;110: 839-846.

14 Andrieux J, Demory JL, Caulier MT, et al. Karyotypic abnormalities in myelofibrosis following polycythemia vera. Cancer Genet Cytogenet 2003;140:118-123.

15 Rumi E, Harutyunyan A, Elena C, et al. Identification of genomic aberrations associated with disease transformation by means of high-resolution SNP array analysis in patients with myeloproliferative neoplasm. Am J Hematol 2011;86:974-979.

16 Cervantes F, Pereira A. Prognostication in primary myelofibrosis. Curr Hematol Malig Rep 2012;7:43-49.

17 Passamonti F, Rumi E, Caramella M, et al. A dynamic prognostic model to predict survival in postpolycythemia vera myelofibrosis. Blood 2008;111: 3383-3387.

18 Barosi G. Emerging targeted therapies in myelofibrosis. Expert Rev Hematol 2012;5:313-324.

19 Cervantes F, Dupriez B, Passamonti F, et al. Improving survival trends in primary myelofibrosis: an international study. J Clin Oncol 2012;30:2981-2987. 